\title{
Cancer and the Levels of Selection
}

\author{
Samir Okasha
}

\begin{abstract}
Cancer is often seen as a case of multilevel selection, in which selfish cancer cells pursue short-term proliferation to the detriment of the collective. Thus cancer cells are described as 'cheats', and an analogy is often drawn between the mechanisms by which organisms fight cancer and the mechanisms by which social groups enforce cooperation. Recently, Gardner ([2015a]) and Shpak and Lu ([2016]) have argued that cancer is not a true case of multilevel selection, that cancer cells should be not regarded as cheats, and that the analogy between anticancer adaptations and suppression mechanisms in social groups is misleading. Their arguments are powerful. However, by drawing on the (presumed) link between cancer and the evolution of multicellularity, the notion that cancer represents a form of selective conflict between cells and organisms can be salvaged.
\end{abstract}

1 Introduction

$2 \quad$ Evolution and Cancer

$3 \quad$ The Evolutionary Dead-End Argument

3.1 Gardner ([2015a], [2015b])

3.2 Shpak and Lu ([2016])

3.3 Further considerations on the EDA

3.4 Upshot so far

$4 \quad$ Cancer and the Origins of Multicellularity

4.1 Buss on germ-line sequestration

4.2 Cancer as atavism

5 Conclusion 


\section{Introduction}

According to Wikipedia, 'cancer is a classic example of what evolutionary biologists call multilevel selection'. ${ }^{2}$ The article goes on to explain that since cancer is usually fatal for an individual organism, 'there is selection for genes and the organization of tissues that suppress cancer; but since cancerous cells typically proliferate faster than other somatic cells within an organism,'at the level of the cell there is selection for cancer'. The suggestion, then, is that cancer is an instantiation of a well-known evolutionary scenario, in which natural selection operates at more than one level of a hierarchically-organized system. Such scenarios are the subject matter of 'multilevel selection theory', a branch of evolutionary biology with diverse applications. ${ }^{2}$ In a typical multilevel scenario, replicating 'particles' are nested within 'collectives', which often leads to evolutionary conflict. For a trait that is advantageous at the particle level may be disadvantageous at the collective level or vice-versa, in which case the direction of selection will be different at each level. In the case of cancer, the levels in question are the cellular and the organismic; the suggestion is that cancerous cell lineages, by escaping the normal constraints on cell division, enjoy a short-term selective advantage despite long-term detriment to the organism.

It is true that many biologists see cancer as a case of multilevel selection, just as Wikipedia says. ${ }^{3}$ Indicative of this is the widespread tendency to apply concepts and descriptors from social evolution theory (of which multilevel selection is a part) to cancer. Thus for example, malignant cells in a neoplasm are described as 'selfish' or 'cheaters', seeking to benefit themselves at the expense of the collective, by contrast with the 'cooperative' behavior of normal somatic cells. ${ }^{4}$ The language of selfishness, cheating and cooperation has its original home in the study of animal social behaviour, but in principle applies whenever there is conflict between levels of selection. The same is true of the term 'policing mechanism', which in the case of cancer has been used to describe the means by which organisms try to prevent cancerous

\footnotetext{
${ }^{1}$ 'Somatic evolution in cancer' in Wikipedia, The Free Encyclopedia.

${ }^{2}$ For discussions of multilevel selection, see (Sober and Wilson [1998]; Frank [1998]; Gould [2002]; Okasha [2006]; Godfrey-Smith [2009]; Goodnight [2013]; Gardner [2015a]).

${ }^{3}$ Examples include (Buss [1987]; Greaves [2015]; Aktipis and Nesse [2013]; Aktipis [2020]; Maley and Reid [2005]; Goodnight [2013]).

${ }^{4}$ Cancer is described in these terms by (Burt and Trivers [2006], Chapter 11; Ratcliff et al. [2012]; Nelson and Masel [2017]; Aktipis and Maley [2017]; Inglis et al. [2017]).
} 
cells from arising or spreading, such as programmed cell death and immune system destruction of nascent tumours. This invites an analogy with the policing that goes on in social insect colonies, for example, when workers eat the eggs laid by other workers to ensure that only the queen's progeny are reared. ${ }^{5}$ When cancer is described in these terms, then even if the multilevel selection framework is not explicit, it is effectively presupposed.

The tendency to view cancer in this way, as the result of conflict between levels of selection, is manifest not just in the language biologists use but also in the explanations they give. For example, it is often argued that the abnormal behaviour of cancer cells, though puzzling at first sight, becomes explicable once we realize that they differ genetically from normal somatic cells, thanks to mutations and / or chromosomal re-arrangements. ${ }^{6}$ According to this argument, the cooperation of the cells in an organism is the default expectation, given that they derive from a single zygote, but cancerous cells, being mutants, are exceptions. This argument invokes one of the central principles of social evolution theory, namely that clonally related units have identical evolutionary interests so will be selected to cooperate, while genetically different units usually will not.

In recent articles, Gardner ([2015a], [2015b]) and Shpak and Lu ([2016]) both reject the idea that cancer represents a bona fide case of multilevel selection, on the grounds that cancer cells are an evolutionary dead-end. Gardner observes that a cancerous cell lineage in an organism dies out when its host organism dies, apart from in the extremely rare cases of transmissible tumours (only two such cases are known. ${ }^{7}$ ) So despite its short-term proliferative advantage, a cancer cell leaves no descendants in the next organismic generation, so makes no contribution to the future of the gene pool. Thus the idea that there is selection for cancer at the cell level, but against it at the individual level, is flawed, Gardner argues. Shpak and Lu ([2016]) endorse Gardner's critique; in addition, they argue that cancer is

\footnotetext{
${ }^{5}$ This analogy is explicit in (Aktipis [2020]; Aktipis and Maley [2017]; Goodnight [2013]).

${ }^{6}$ For example, Crespi and Summers ([2005]) write 'cells within the metazoan body are, for the most part, genetically identical; thus, they have evolved an altruistic division of labor represented by diverse, specialized and integrated types of tissue. By contrast, the somatic selection of cancer is driven by differential replication of cells that differ phenotypically as a result of genetic mutation and epigenetic alteration (p. 545)'.

${ }^{7}$ These occur in the domestic dog (Canis lupus familiaris) and the Tasmanian devil (Sarcophilus harrisii) (Murchison [2008]). Shpak and Lu ([2016]) also mention heritable tumourigenic mutations in plants.
} 
fundamentally dissimilar from paradigm cases of multilevel selection, as for example a transposon that reduces its host organism's fitness but spreads by inserting multiple copies of itself in the genome. Thus biologists are wrong to see cancer 'as a case study in conflict between two classes of replicating entities - cells and organisms', they argue (p. 42). This leads Shpak and Lu to reject the idea that cancer cells are usefully thought of as 'cheaters', and to reject as misleading the analogy between the mechanisms by which organisms suppress cancer and the policing mechanisms found in social groups for suppressing defectors.

This evolutionary dead-end argument - hereafter the EDA — poses a prima facie challenge to the view that cancer involves conflict between levels of selection. Do proponents of that view - who of course know that cancer is usually non-transmissible - have the wherewithal to respond to the challenge? And if the EDA is decisive, then what exactly needs to change? Merely the language that some biologists have used to describe cancer, or something more substantive? These are the questions that I tackle in this paper.

I think of this as a quintessentially philosophy-of-science matter. For the disagreement is not, or not primarily, about how and why cancer occurs, but rather about the validity of a certain conceptual framework for thinking about cancer. Thus the key question is not of the form 'what are the empirical facts?', but rather 'what would the empirical facts have to be like, in order for the levels-of-selection framework to be applicable?'. The answer that Gardner and Shpak and Lu give is that cancer would have to be transmissible - as it usually is not. But there are other possible answers too, some of which seem to be implicitly held by those who conceptualize cancer in multilevel selection terms. They include: (i) that the clonal evolution model of carcinogenesis would have to be true; (ii) that the theory of Buss ([1987]) on the origins of multicellularity would have to be true; and (iii) that the so-called 'atavistic interpretation' of cancer would have to be true. Each of (i)-(iii) constitutes a potential response to the EDA and needs to be carefully evaluated.

The structure of the paper is as follows. Section 2 distinguishes the multilevel selection view of cancer from a number of other ways in which evolutionary ideas have been brought to bear on cancer, including the clonal evolution model. Section 3 considers the EDA in more detail and discusses some possible responses. Section 4 deals with the relation between cancer and the evolution of multicellularity. Buss's theory and the atavistic interpretation are discussed, and their potential to deal with the challenge posed by the EDA is assessed. Section 5 concludes. 


\section{Evolution and Cancer}

That evolution can shed light on cancer is uncontroversial. In fact, three distinct ways of applying evolutionary principles to cancer can be distinguished, each of which has led to insights. Firstly, evolutionary theory can be used to explain the population-wide incidence of inherited germ-line mutations that are known to increase cancer risk, such as the $B R A C 1$ gene implicated in breast cancer. Since such mutations are harmful for organisms, the challenge is to explain why natural selection has not eliminated them. Possible answers include recurrent mutation; antagonistic pleiotropy (a mutation having two opposing effects on fitness); a mismatch between ancestral and modern environments (such as fair-skinned people living in sunny climates); and others. Note that the type of natural selection at work, when Darwinian evolution is invoked in this context, is 'ordinary' selection between individual organisms based on their heritable traits, in this case propensity to develop cancer. That selection of this sort occurs is beyond doubt; but it is of fairly limited significance in explaining the prevalence of cancer, given that many more cancers result from somatic mutations and / or epigenetic changes than from germ-line mutations.

Secondly, evolution can be used to understand the various adaptations that organisms have for preventing cancer from arising or spreading. These include tumour suppression mechanisms such as DNA repair, apoptosis, and regulation of cell division; inhibition of metastasis via cell adhesion; immune system destruction of cancer cells; tissue architecture that impedes the spread of cancerous cells, such as colonic crypts; a reduced somatic mutation rate in stem cells, for example via non-random DNA strand segregation ${ }^{8}$; and more. The evolutionary logic here is again straightforward. Cancer, whatever its proximate cause, is a major source of mortality; and if it strikes before reproductive age, constitutes a powerful selective pressure. So we should expect organisms to evolve adaptations to combat it. When invoked in this context, Darwinian evolution is playing its standard explanatory role, namely explaining certain organismic attributes in terms of their adaptive significance, namely cancer prevention.

\footnotetext{
${ }^{8}$ This is the idea, first advanced by Cairns ([1975]), that dividing stem cells do not segregate DNA strands randomly into daughter cells but preferentially allocate the (ancestral) template strand to the daughter stem cells. This potentially reduces the rate of mutation accumulation in tissues. See (Werner and Sottoriva [2018]) for a recent discussion.
} 
Thirdly, and in sharp contradistinction to the above, there is the idea that tumour development itself is an evolutionary process, involving selective competition between cell lineages within the lifespan of a single organism. This idea, propounded in particular by Nowell ([1976]), is now widely accepted in both the medical and evolutionary literatures, where it is often referred to as the 'clonal evolution' or 'somatic evolution' model of cancer. ${ }^{9}$ The basic idea is that a neoplasm can be thought of as a large population of heterogeneous cells, differing both genetically and epigenetically, some of which are better at surviving and reproducing than others. This cellular population meets the basic requirements for Darwinian evolution - variation, heritability and fitness differences - where 'fitness' here refers to cell fitness, as measured by proliferation rate. Thus faster-dividing cells outcompete their rivals within the neoplasm, leading to clonal expansion. (Neutral processes may play a role too.) Further mutations then arise, providing more fuel for selection, and the process repeats, leading to progressive development of a tumour.

The clonal evolution view of cancer is no mere superficial application of Darwinism. On the contrary, it has genuine explanatory power. ${ }^{10}$ All of the famous 'hallmarks of cancer' identified by Hanahan and Weinberg ([2000], [2011]), such as resistance to apoptosis, inducing angiogenesis and evading growth suppressors, can be viewed as complex adaptations that cancer cells have evolved (somatically) via multiple rounds of mutation and selection, as a number of authors have noted. ${ }^{11}$ Thus a Darwinian perspective allows us to understand these cellular phenotypes in terms of their adaptive significance at the cell level. Moreover, the clonal evolution view helps explains why tumours invariably evolve resistance to cytotoxic chemotherapy. The explanation is that the introduction of chemotherapetic drugs alters the selective microenvironment, so when mutation throws up a resistant cell it will undergo rapid clonal expansion. As is well known, this is similar to how bacteria evolve resistance to antibiotics, which is a bona fide Darwinian process. And finally, phylogenetic studies of tumour development, that study the pattern of descent of the cell clones within a single tumour, have recently flourished; this underscores the fact that tumourigenesis is a genuinely evo-

\footnotetext{
${ }^{9}$ Morange ([2012]) notes that the idea in fact has a considerably older pedigree, tracing to the work of Boveri ([1914]).

${ }^{10}$ Though see (Germain [2012]) for an interesting argument to the contrary, based on Godfrey-Smith's ([2009]) notion of a 'marginal Darwinian population'.

${ }^{11}$ This argument is found in (Merlo et al. [2006]; Greaves [2015]; Lean and Plutynski [2016]; Fortunato et al. [2017]), among others.
} 
lutionary phenomenon. ${ }^{12}$

Recently, the clonal evolution model has been contrasted with an alternative model of carcinogensis known as the cancer stem cell (CSC) model (Germain [2012]; Laplane [2018]). According to the CSC model, cancer cells fall into two distinct sub-populations: CSCs, which have effectively unlimited proliferative ability, and non-CSCs, which can divide at most a handful of times before cell death. So only those mutations that arise in CSCs can give rise to a clonal population that will persist. On the CSC model, Darwinian competition between rival sub-clones in a tumour plays a less important role, since most sub-clones will die out anyway. The empirical validity of the CSC model is a topic of ongoing research, but even if it is validated, this would not disprove the clonal evolution model outright (Plutynski [2020]). For the two models represent ends of a continuum, not hard-and-fast alternatives, and it seems probable that different cancers will lie at different points on that continuum.

I assume, then, that the clonal evolution model of cancer is empirically well-founded. But importantly, this does not in itself vindicate the idea that cancer is a case of multilevel selection. For on the clonal evolution view, the cell-level selection is between variant cancer cell lineages within a neoplasm. It is this that explains why malignant cells acquire the hallmarks of cancer, develop resistance to chemotherapy, and why phylogenetic analysis works. But on the multilevel selection view, cancer is depicted as a conflict between selection at the levels of the cell and the organism; so the relevant form of within-organism selection would have to be between cancerous cell lineages and normal somatic cell lineages, which is a different matter. That selection of this latter sort occurs is not a crazy proposition. After all, we know that within the tumour microenvironment, cancerous cells do compete with neighbouring somatic cells, by diverting resources away from them and coopting their activities for their own purposes (for example, hijacking blood vessels). Cell-lineage competition of this sort could be (and sometimes is) interpreted as a form of Darwinian selection, in which cancer cells are the 'winners' and somatic cells the 'losers'. ${ }^{13}$ But crucially, such competition involves only the small subset of somatic cells that interact directly with cancer cells. The existence of such competition, therefore, does not vindicate

\footnotetext{
${ }^{12}$ See (Schwartz and Schäffer [2017]) for a review of cancer phylogenetics.

${ }^{13}$ Cell competition is described in Darwinian terms in (Moreno and Rhiner [2014]) and (Claveria and Torres [2016]).
} 
the notion of a ubiquitous evolutionary conflict between cancerous cells and normal somatic cells, for that notion would only apply if there was a bona fide Darwinian selection process acting on the total population of cells in the organism. Perhaps it is defensible to envisage such a process. We return to this issue later; for the moment the point is just that the empirical success of the clonal evolution model does not settle the matter.

At this juncture, it is worth noting that advocates of the clonal evolution model have sometimes invoked multilevel selection in a quite different way from that which is our main concern here. Two examples will illustrate. Firstly, Aktipis et al. ([2015]) argue that in advanced metastatic cancer, there is often a metapopulation of genetically distinct cell colonies (or clones); and that the resulting population structure can favour cooperation. ${ }^{14}$ This argument invokes the well-known principle, integral to multilevel selection theory, that grouping of individuals can facilitate the evolution of cooperative behaviours that could not evolve in an unstructured population. The suggestion is that this principle, when applied to the cancer cells within a neoplasm, might explain the empirical observation that some cell clones appear to cooperate with each other in tumour formation, rather than exhibiting purely selfish behaviour. Note that in this application of multilevel selection, the particles are the individual cancer cells and the collectives are colonies (or clonal groups) of such cells, not organisms. That is, multilevel selection is here invoked as part of the clonal evolution model's explanation of tumourigenesis. This is quite different from the idea that cancer involves evolutionary conflict between cells and organisms.

Secondly, Lean and Plutynski ([2016]) also invoke multilevel selection in the context of the clonal evolution model. Their argument is that cancer progression should not be regarded as a purely individual-level selective process; rather, group selection offers a useful perspective too-where a 'group' means a group of cancerous cells, or a whole tumour. Their reasons for saying this are various; they include the idea that genetically distinct cancer cell lineages can co-evolve with each other; that population structure within a tumour could affect the outcome of somatic evolution (as per Aktipis' suggestion above); that properties of a tumour as a whole could affect the likelihood of it invading other tissues; and that metastasis can be regarded as 'reproduction by tumours'. These are interesting ideas; here is not the place to

\footnotetext{
${ }^{14}$ Similar arguments are found in (Cleary et al. [2014]; Axelrod et al. [2006]).
} 
assess their empirical validity. ${ }^{15}$ The key point to note is that when multilevel selection is invoked in this way, it refers to a process that takes place entirely within a single organism during the course of tumour progression; this is very different from multilevel selection in the sense of a combination of selection between cells within organisms and selection between organisms, which is the target of the EDA.

To summarize, there are three uncontroversial ways in which evolutionary principles can be applied to cancer: (i) to explain the incidence of germ-line mutations that increase cancer risk; (ii) to explain why organisms evolved anti-cancer adaptations; (iii) to explain the process of tumour development via cell-lineage selection, as in the clonal evolution model. However points (i)-(iii) do not in themselves vindicate the idea that cancer involves conflict between levels of selection.

\section{The Evolutionary Dead-End Argument}

The EDA says that it is mistaken to conceptualize cancer in terms of conflict between levels of selection, since cancer cells are not transmitted to future organismic generations (except in very rare cases). Gardner's and Shpak and Lu's defences of this argument are examined below. But firstly, one point about non-tranmission should be noted. That cancer cells are not transmitted is a highly contingent fact, not an intrinsic limitation, in the sense that many cancer cells do possess indefinite replicative potential - unlike ordinary somatic cells in metazoan tissue - so can in principle outlive their hosts. This point is stressed by Vincent $([2010])$, who observes that cancer cells 'can regularly be cultured from host biopsies, propagated in vitro and even transplanted serially into immunologically tolerant recipients, such as nude mice, in the form of xenografts' (p. 1177). Thus strictly speaking, we should say that cancer cells are de facto not transmitted, rather than that they are non-transmissible.

\subsection{Gardner ([2015a], [2015b])}

Gardner's version of the EDA derives from his 'genetical theory of multilevel

\footnotetext{
${ }^{15}$ See (Germain and Laplane [2017]) for critical discussion of Lean and Plutynski's use of multilevel selection, and (Plutynski [2018]) for further discussion.
} 
selection'. This is a general framework that Gardner develops for analysing evolution in hierarchically structured systems, based around the Fisherian concept of reproductive value (Fisher [1930]). The latter is a mathematical measure of the long-term evolutionary success of an allele, genotype, or organism (roughly, its expected contribution to the future gene pool of the population or species). Since all somatic cells, including cancer cells, die when their host organism dies, these cells have zero reproductive value, Gardner argues. So the proliferation of cancer cells within an organism does not represent 'selection in the strict sense' (p. 6); thus cancer should not be conceptualized as a form of conflict between levels of selection.

What should we make of this argument? In Gardner's defence, applying the concept of reproductive value to multilevel selection is perfectly defensible. The main point of that concept is to supply a fitness measure that takes account of the fact that individuals in a population can vary in non-genetic ways, so fall into distinct classes (age groups, sexes, castes), which may differ in terms of their contribution to the future gene pool. The existence of class structure means that simply counting offspring is an unsuitable measure of an organism's Darwinian fitness; the distribution of offspring across classes matters too, which reproductive value takes account of. This general point applies to multilevel no less than to single-level scenarios.

However, Gardner's line of argument can be contested in at least three ways. Firstly, certain multilevel selection phenomena arguably cannot be accounted for from a genetic basis anyway, as they involve higher-level changes. Arnold and Fristrup ([1982]) gave a striking example in which selection between species changes the composition of species within a single clade, but without any underlying changes in gene frequency. Though not directly related to the issue of cancer and reproductive value, this point does suggest that Gardner's genetical theory may be unable to capture the full range of evolutionary phenomena which multilevel selection has traditionally been invoked to explain.

Secondly, Gardner's argument, if correct, would seem to condemn not just the idea that cancer involves multilevel selection but also the clonal evolution model of tumourigenesis. For unless we allow that competition between cell lineages within a neoplasm is a bona fide case of natural selection, the empirical success of the clonal evolution model is hard to explain. Now Gardner might reply that the differential proliferation of cancer cells (and somatic selection more generally) is merely 'selection-lite', that is, a selectionlike phenomenon that falls short of being the real thing. (This is suggested 
by Gardner's phrase 'selection in the strict sense', quoted above.) However, this reply seems somewhat arbitrary; for the fundamental requirements for evolution by natural selection - variation, heredity, and fitness differencesare satisfied in cases of somatic selection no less than in cases of 'ordinary' natural selection. ${ }^{16}$

Thirdly, Gardner's argument arguably proves too much, as can be seen by frameshifting it up a level. Since over geological time, the vast majority of species go extinct without leaving any daughter species, the organismal lineages within them leave no long-term descendants either. But the organisms within these species clearly do evolve by natural selection, during the species' lifetimes. Now Gardner might reply that his genetical theory of multilevel selection is inherently microevolutionary, focusing on allele frequency change within a species, and thus that the relevant definition of 'long-term' does not extend beyond a single species' lifetime. But again, if this is right it suggests that Gardner's theory is not a wholly general analysis of multilevel selection. Alternatively, Gardner might reply that it is merely a contingent fact that most species will go extinct, so any given organism may leave descendants in future species. However the force of this reply is dented by the point noted above, that a cancer cell's inability to outsurvive its host is also a contingent limitation, and one that can be lifted artificially.

Where does this leave us? Gardner's argument certainly poses a challenge to those who conceptualize cancer in terms of conflict between levels of selection. However the case is not clear-cut; as there are a number of defensible counterarguments, allied to doubts about the generality of Gardner's conception of multilevel selection. To make progress, let us turn to the version of the EDA found in Shpak and Lu ([2016]). Their argument is a development of Gardner's but is not tied to the notion of reproductive value.

\subsection{Shpak and Lu ([2016])}

According to Shpak and Lu ([2016]), since somatic mutations are an evolutionary dead-end, 'multilevel selection (sensu stricto) on cancer is rarely possible' (p. 42). In support of this, they offer a general principle about what is needed for 'true' multilevel selection. They write: 'reducing the fitness of one class of replicators through selection on replicators at another level of

\footnotetext{
${ }^{16}$ Goodnight ([2015]) objects to Gardner's argument on precisely these grounds; but see the reply by Gardner ([2015b]).
} 
organization is not sufficient for multilevel selection per se. In true multilevel selection, the lower-level replicator has to have the potential to contribute to the genotype or phenotype of the next generation (with respect to the higher-level replicators generation time). This includes the potential to drive genotype frequencies in opposite directions across generations' (p. 42).

Shpak and Lu make an important point here. It is certainly true that in many paradigm cases of multilevel selection, selection at the lower and the higher levels can offset each other over the generation time of the higherlevel unit; and that therein lies much of the interest in this type of selection process. Consider for example the famous case of the $t$-allele in the domestic house mouse (Lewontin and Dunn [1960]). This allele is a selfish genetic element which spreads at the expense of its host organism. Mice homozygotic $(t / t)$ for the allele die before reproducing; so at the level of the organism the allele is selected against. But heterozygotes $(t /+)$ transmit the $t$-allele to $90 \%$ of their gametes, so at the gametic level the allele is selected for. Thus the allele is maintained in the population by the two opposing selective forces. As Shpak and Lu point out, nothing comparable happens in the case of cancer. A mutation (germline or somatic) that causes cancer, and so reduces the individual organism's survival, cannot be maintained in a population by the countervailing force of lower-level (somatic) selection; for although the mutation may proliferate somatically within an organism, it is not transmitted to the next (organismic) generation.

Shpak and Lu illustrate this point formally using the Price equation, a standard tool for modeling multilevel selection. ${ }^{17}$ Letting $\bar{z}$ denote the frequency of a gene in a population of organisms, the change in frequency over a single (organismic) generation can be written as $\Delta \bar{z}=\operatorname{Cov}(w, z) / \bar{w}+$ $\operatorname{Exp}(w \delta) / \bar{w}$, where $w$ denotes fitness, $\bar{w}$ is average fitness, and $\delta$ measures parent-offspring deviation in genotype. The Cov term is the covariance, across individuals in the population, between fitness and genotype, so captures the effects of selection at the organismic level; while the Exp term captures the changes arising from imperfect transmission, including lowerlevel selection. Applied to the $t$-allele in mice, the Cov term is negative but this is offset by a positive Exp term, thanks to gametic selection. But applied to a gene associated with cancer, matters are different. In this case, the lower-level (somatic) selection, that is, the proliferation of somatic cells

\footnotetext{
${ }^{17}$ See (Price [1972]; Frank [1998]; Okasha [2006]) for discussion of the Price equation and multilevel selection.
} 
containing the gene within an individual organism, will have no effect on the Exp term, since somatic cells are not transmitted to the next generation.

This is an interesting argument. One possible response is to contest Shpak and Lu's criterion for 'true' multilevel selection. After all, multilevel selection is a broad concept with a rather convoluted history, lacking a canonical formulation that all parties agree on. ${ }^{18}$ In the macroevolutionary literature on species and clade selection, for example, multilevel selection is usually taken to mean the combination of selection on organisms within species and differential extinction of species, processes which take place over very different timescales ${ }^{19}$; so the effects of the lower-level selection are not necessarily manifest over the generation time of the higher-level unit. In such cases Shpak and Lu's criterion will not always be satisfied.

How might Shpak and Lu reply? Conceivably, they could appeal to the distinction between: (i) selection at multiple levels of a hierarchical system contributing to a single evolutionary change (for example, change in allele frequency across organismic generations); and (ii) selection at multiple levels of a hierarchical system each leading to a different evolutionary change, possibly with some causal interaction between them. The former involves an evolutionary process driven by selection at more than one level; while the latter involves a multiplicity of evolutionary processes, at different levels, each driven by selection at its own level. More succinctly, (i) involves a single process of multilevel selection, while (ii) involves multiple processes of single-level selection (occurring at different levels). There is an important logical difference here, however we wish to mark it. ${ }^{20}$ It is open to Shpak and $\mathrm{Lu}$ to insist that multilevel selection of the sort that they are concerned with is of type (i); and here their criterion is applicable.

However, once the debate has gone in the direction, the whole issue may start to seem terminological, about how the term 'multilevel selection' should be used. But in fact Shpak and Lu's argument (and Gardner's) raises deeper issues than this. In particular, is the organizing framework of social evolution theory, including the explanations that it offers of selfishness, cheating,

\footnotetext{
${ }^{18}$ Compare the substantially different conceptions of multilevel selection found in (Sober and Wilson [1998]; Frank [1998]; Okasha [2006]; Gould [2002]; Damuth and Heisler [1988]; Gardner [2015a]; Goodnight [2013]).

${ }^{19}$ See, for example, Brunet and Dootlittle ([2015]).

${ }^{20}$ The distinction between (i) and (ii) is in effect equivalent to the distinction between 'MLS1' and 'MLS2' introduced by Damuth and Heisler ([1988]) and elaborated by Okasha $([2006])$.
} 
cooperation and conflict, applicable to cancer? Is the conflict between cancerous cells and the organism relevantly similar to other conflicts between levels of selection? Is the analogy between suppression of cheats in social groups and organisms' anti-cancer adaptations, that many authors endorse, a valid one? These are important conceptual questions, not to be resolved by terminological fiat. It seems preferable, therefore, to set aside the issue

of what constitutes 'true' multilevel selection and to focus directly on these questions.

Now the EDA strongly suggests that the answer to each of these questions is 'no'. For a corollary of Shpak and Lu's point is the following. The sense in which a selfish genetic element such as the $t$-allele is 'selfish', and the sense in which a cancer cell (or a mutant gene within it) is 'selfish', are fundamentally different. That is, although both the $t$-allele and the mutant cancer cell can be said to benefit themselves at the expense of the organism, the meaning of 'benefit' and the currency in which it is measured are different in the two cases. In the former case, the currency is number of copies left in the next organismic generation; in the latter, it is number of copies left in the organism's somatic tissues (or rate of somatic proliferation). Thus it does indeed seem misleading to think of cancer cells as selfish cheats, just as Shpak and $\mathrm{Lu}$ argue. Further considerations in support of this are introduced below.

\subsection{Further considerations on the EDA}

There is increasing evidence that somatic mutations not only cause cancer, but also other diseases that do not involve malignancy, including primary immune deficiencies, hypertension, and various neurological disorders. ${ }^{21}$ Such diseases are clearly harmful to the individual, just like cancer. Now if a cancer cell is regarded as 'selfish' on the grounds that it benefits itself but harms the organism, by parity of reasoning a somatic cell that causes nonneoplastic disease should be regarded as 'spiteful', since it harms the organism but does not benefit from an enhanced proliferation rate. ('Spite' is the standard term in social evolution for a behaviour which imposes a fitness cost on both actor and recipient.) Furthermore, since selection can work on epigenetic as well as genetic variation, any somatic cell in healthy tissue that is terminally differentiated could be regarded as 'altruistic': it has sacrificed its own proliferation in order to perform somatic duties for the organism's

\footnotetext{
${ }^{21} \mathrm{See}$ (Li and Williams [2013]; Leija-Salazar et al. [2018]) for overviews.
} 
benefit. Similarly, a B cell in the immune system that undergoes clonal expansion in response to an antigen can be regarded as 'cooperative', for it enjoys enhanced proliferation and also benefits the organism. The oddity of these conceptualizations, and the fact that the terms 'spite', 'altruism' and 'cooperation' are not generally applied in this way, should give further pause to those who regard cancer as a form of cellular selfishness.

This consideration ties in with the point made in section 2 , that the validity of the clonal evolution model cannot be used to rebut the EDA. We noted that the clonal evolution model owes its explanatory success to its positing a selective process between mutant cell lines within a neoplasm, not between cancerous cells and other somatic cells within the whole organism. But it is a selective process of the latter sort that would be needed to vindicate the notion that cancerous cells are 'selfish', pursuing their own interests at the expense of the rest of the soma. For this to make sense, we would need to regard all the cells in a single organism as comprising a single population undergoing selection, in which a cell's 'fitness' is measured by its rate of mitotic division. ${ }^{22}$ But there seems no good reason to think in these terms, not least because differences in mitotic division rates are (usually) under genomic control. As noted above, there are many genetically and epigenetically-based differences in somatic cell division rates that are not naturally interpreted as a form of Darwinian selection, nor described in terms of social evolution concepts. So the fact that cancer cells proliferate faster than normal cells, and thereby harm the organism, does not vindicate the idea that there is a bona fide Darwinian selection process occurring on the total population of cells within an organism in which 'fitter' cancer cells outcompete 'less fit' somatic

\footnotetext{
${ }^{22}$ Here I concur with Godfrey-Smith ([2009]), who argues that the cells in a multicelled organism do not form a 'paradigm Darwinian population', but for quite different reasons (p. 56, pp. 102-3). Godfrey-Smith's reason for saying this is that which cells in an embryo become germ cells rather than somatic cells depends on position rather than intrinsic cell character. This violates what Godfrey-Smith regards as a prerequisite for any set of entities to form a Darwinian population, namely that fitness differences should depend on intrinsic differences. Note that Godfrey-Smith is arguing against a putative application of Darwinism to the population of cells in an organism in which the germ cells have high fitness and the somatic cells low fitness - that is, in which cellular fitness is measured by number of descendants in the next organismic generation. By contrast, I am arguing against a putative application of Darwinism to the population of cells in an organism in which the fittest cells are those with the highest somatic proliferation rate within the organism. This latter application is the one that would have to be defensible, to vindicate the idea that cancer cells are 'selfish' and somatic cells 'altruistic'.
} 
cells.

This point illustrates the fact that the EDA has consequences not just for how cancer is described but also for how it is explained. Recall the claim that the behaviour of cancer cells is readily explicable given that they differ genetically from normal cells. This oft-made claim is actually ambiguous as between a proximate and an ultimate reading. On the proximate reading, the claim is that the proximate explanation of why cancer cells proliferate uncontrollably is that they harbour genetic mutations, which is unquestionably true. But on the ultimate reading, the claim is that since cancer cells are genetic mutants, their evolutionary interests no longer coincide with those of the other cells in the organism, so we should expect them to abandon normal somatic duties in favour of self-interested pursuits. Understood this way, the claim relies on a social evolution principle, namely that coooperation is the default expectation where entites are genetically identical but not otherwise. But if the EDA is right, this social evolution principle has no relevance for cancer. If it is wrong to think of cancer as a form of selfish cheating, then we cannot regard uncontrolled proliferation as an adaptive strategy that 'makes sense' for a cancer cell; so the fact that the cancer cell is a mutant does not allow us to explain, in the sense of supply an evolutionary rationale for, its behaviour; thus on the ultimate reading, the claim fails unless the EDA can be successfully rebutted.

\subsection{Upshot so far}

Gardner and Shpak and Lu offer powerful arguments against the idea that cancer involves multilevel selection. However their arguments are not decisive, in part because they rely on conceptions of multilevel selection that are contestable. To avoid the debate becoming semantic, it is best to sidestep the issue of what 'true' multilevel selection is. Instead, we may construe the EDA as an argument against viewing cancer as a form of cellular cheating, and/or explaining it in terms of evolutionary conflict between cell and organism, and/or conceptualizing it in the framework of social evolution theory. So construed, the argument is a powerful one, and poses a serious challenge to these biologists who understand cancer in these terms. I turn next to what I regard as the most promising way of meeting the challenge, which draws upon the (presumed) link between cancer and the evolution of multicellularity. 


\section{Cancer and the Origins of Multicellularity}

As we have seen, the notion that a mutant cancer cell in a modern organism 'benefits' from the uncontrolled proliferation that it causes is questionable, given that it is an evolutionary dead-end. However, multicelled organisms as we know them today have not always existed. Rather, they evolved gradually from single-celled ancestors - a process that must have involved selection at both the cell and organism levels. ${ }^{23}$ For this transition to multi-cellularity to happen, the cells within an organism had to cooperate with each other, surrender their reproductive rights, and perform somatic duties for the good of the whole. In particular, for cellular differentiation - so crucial to embryonic development - to evolve, some cell lineages had to relinquish totipotency and thus lose the capacity for unlimited proliferation. Organisms evolved a variety of control mechanisms for ensuring that cells perform their somatic duty correctly. Plausibly, cancer results when these mechanisms break down.

This suggests an alternative justification for regarding cancer cells as 'cheats', selfishly benefiting themselves at the expense of the organism. The putative justification lies in facts about the deep evolutionary past, rather than the present. That is, it is not the mere fact that cancer cells proliferate faster than ordinary somatic cells in modern organisms that justifies this conceptualization, but rather the fact that such uncontrolled proliferation represents a reversion to a mode of living found in single-celled ancestors, for whom it was beneficial, and which organisms had to suppress in order for multicellularity to evolve. Similarly, the justification for viewing cancer as a form of selective conflict between cell and organism lies in the fact that such a conflict occurred in the evolutionary past, and that the mechanisms that organisms evolved to control it are precisely those whose breakdown leads to cancer today.

Two versions of this argument are explored below, that rest on different empirical premises. The first draws on ideas from Leo Buss's The Evolution of Individuality (Buss [1987]). The second draws on the idea, popular in the recent cancer literature, that neoplastic cells are atavistic, that is, have reverted to an ancestral state. Both represent possible ways of justifying the view that cancer involves cheating, and conflict between levels of selection, despite cancer cells being an evolutionary dead-end.

\footnotetext{
${ }^{23}$ This is a prominent theme in the work of Buss ([1987]) and Michod ([1999], [2007]).
} 


\subsection{Buss on germ-line sequestration}

Buss's 1987 book played a key role in the genesis of the literature on evolutionary transitions, which studies how free-living biological units become aggregated into larger units. ${ }^{24}$ Buss's main focus was on the transition from single-celled to multi-celled organisms (which he called 'individuals'). He advocated a levels-of-selection perspective, arguing that to understand the transition in Darwinian terms, we need to invoke selection at both the cell and organism levels, and the tension between them. Numerous aspects of embryonic development in modern metazoa, Buss argued, bear testimony to the ancestral conflict between selective levels.

One of Buss's key claims was that during the evolution of multicellularity, organisms faced a continual threat from 'rogue' cell lines that pursued their own interests at the expense of the organism. He identified two organismic features that serve to contain this threat: maternal control of early development and germ-line sequestration. The former refers to the fact that the earliest stages of embryonic cell division are directed by the maternal genotype, thus limiting the potential for mutant cell lines to disrupt development. The latter refers to the fact that in most metazoan taxa, the future germ cells are set aside, or sequestered, in early ontogeny. This is not a universal feature of multicellular life; plants do not sequester a germline, with many tissues retaining the ability to give rise to a new plant. ${ }^{25}$ Buss argued that animals evolved germ-line sequestration in order to limit the heritability of selfish somatic mutants, that is, to prevent them from invading the germ line. Plants had no need for this given that their rigid cell walls limit the migration of cells within the organism.

The significance of this is the follows. If Buss's hypothesis about the evolution of germ-line sequestration is correct, then it follows that the nontransmission of somatic mutants is itself an adaptation, an evolved mechanism to protect the integrity of the organism. And of course it is this non-transmission that accounts for the fact that cancerous cell lineages are evolutionary dead ends, incapable of passing on their mutated genomes to

\footnotetext{
${ }^{24}$ Other key works in this literature include (Maynard Smith and Szathmáry [1995]) and (Michod [1999]), both of which were influenced by (Buss [1987]).

${ }^{25}$ Though see (Lanfear [2018]) for a recent re-evaluation of the claim that there is no germ-line sequestration in plants.
} 
the next (organismic) generation. So if Buss is right, then the very feature that, according to the EDA, makes it inappropriate to think of cancerous cells as selfish cheats, is itself the result of an organismic adaptation that evolved to prevent somatic cells from invading the germ-line.

This suggests that if we take an expanded evolutionary horizon, conceptualizing cancerous cells as 'cheats' in conflict with their host organism makes a certain amount of sense. For although cancer cells do not in fact reap any long-term evolutionary benefit from the uncontrolled proliferation they cause, if Buss is right then their behaviour is similar to that of the germline invaders that threatened the earliest multicelled organisms, to which organisms evolved germ-line sequestration in response. These ancient germline invaders were genuine cheats, and were 'selfish' in precisely the sense that selfish genetic elements are, since they were not evolutionary dead-ends; that is, they did (when successful) reap a long-term advantage from their behaviour. So the fact, if it is one, that cancer cells in modern organisms behave similarly to these germ-line invaders justifies conceptualizing them as cheats too.

It follows that if Buss's theory is right, there is a rational response to the EDA. The multilevel selection view of cancer is justified, despite the fact that cancer cells are not transmitted, because the mechanism by which their non-transmission is ensured - germ-line sequestration-itself arose as a means to prevent selective conflict between organisms and their constituent cells. Since the mechanism works almost perfectly (in those taxa in which it is found), actual selective conflict between cells and organisms is today a rarity. But mutant cells continually arise that exhibit the phenotypic features abandonment of somatic function and unlimited proliferation-that selected for germ-line sequestration in the first place. Such cells are therefore akin to selfish cheats, despite not reaping any long-term benefit from their behaviour, since they behave in a way that would have brought such a benefit had it not been for the evolved mechanism that prevents it from being realized. Thus there is a principled reason for conceptualizing cancer in terms of conflict between levels of selection, that does not stem merely from differences in somatic proliferation rate.

Here it is worth noting the distinction, emphasized by Okasha ([2005], [2006]), between 'synchronic' and 'diachronic' approaches to the levels of selection issue. The former concerns selection and adaptation at pre-existing hierarchical levels, while the latter concerns the evolution of the biological hierarchy itself. In critiquing the view that cancer involves multilevel selection, 
both Gardner and Shpak and Lu adopt a synchronic approach: their point is that in organisms as they are now, somatic mutants are not transmitted. This is of course true. However, a valid question is how organisms came to be like this in the first place, that is, how they evolved those features that prevent the transmission of somatic mutants. This is to adopt a diachronic approach to the levels of selection - for in effect, it is to ask how it came to be that cell-lineage selection was prevented from having long-term evolutionary consequences, thus allowing organism-level selection to predominate. Buss's theory is an attempt to answer this question; to the extent that it is successful, it vindicates the use of the mutlilevel selection framework, understood diachronically, as an organizing tool with which to think about cancer.

If Buss's theory on the origin of germ-line sequestration is true, the EDA can thus be rebutted. But is Buss's theory true? This is a controversial issue on which no agreement exists, but a couple of points can be made. Firstly, it is fairly clear that germline sequestration is an evolved attribute, for it is not found in basal metazoan taxa (sponges and corals). Secondly, theoretical models of the evolution of multicellularity suggest that Buss's argument could work. In particular, Michod and Roze ([1999]) have modeled the origins of germ-soma separation. In one of their models, organisms initially reproduce by sending out a propagule containing a random sample of the cells in the adult. They show that if mutants arise that abandon somatic duties, this selects for sequestration of the germ-line - as by preventing most cells from entering the pool that gives rise to the next generation, organisms can reduce the chance that harmful somatic mutants will be passed on. This suggests that suppression of cell-lineage competition could indeed be the reason why germline sequestration evolved, just as Buss argued.

Against this, both Maynard Smith ([1989]) and Queller ([2000]) have argued against Buss's theory, on the grounds that cell-lineage competition can be largely eliminated if the organism's lifecycle passes through a single-celled bottleneck - as in most metazoan taxa - since this ensures clonality of the cells in the organism. Thus the function of germ-line sequestration lies elsewhere, they argue, perhaps in protecting the germ cells from damage. Also, Queller and Strassmann ([2013]) have argued that germ-line sequestration may simply arise as a side-effect of some cell lineages altruistically adopting somatic roles and undergoing differentiation, that is, the germ cells are the ones left over. So no process of active exclusion of cells from the germline need be posited, they argue. The jury is still out on this issue.

Finally, a complication should be noted. Buss's book contains a num- 
ber of argumentative threads, intricately interwoven. Often, he does not sharply distinguish between two ideas. The first is that in the early evolution of multicellularity, organismic integrity was threatened by somatic variants that abandoned somatic duty by failing to undergo terminal differentiation and so enjoying an increased proliferation rate. The second is that organismic integrity was threatened by somatic variants that tried to invade the germline. ${ }^{26}$ These ideas are not equivalent, since the sense in which the somatic variants 'benefit' is different in the two cases (short-term proliferation versus increased frequency in the next organismic generation). The former idea is fairly uncontroversial; it explains why organisms evolved mechanisms to control cell division and anti-proliferative mechanisms such as apoptosis. However it is the latter idea that would need to be true if germline sequestration represents an adaptation designed to limit the heritability of somatic mutants, which is the aspect of Buss's theory that allows a response to the EDA.

To conclude, if Buss's hypothesis on the origin of germline sequestration is true, this entitles us to regard the non-transmission of somatic mutants, including ones that cause cancer, as itself an adaptation designed to limit the effects of selective conflict between cell and organism. This in turn justifies conceptualizing cancer in terms of multilevel selection and / or social evolution, and thus meets the challenge posed by the EDA. However, since it is unknown whether Buss's hypothesis is true, there is a considerable empirical hostage-to-fortune here.

\subsection{Cancer as atavism}

According to the atavistic interpretation, cancer does not represent a novel phenotype, nor the result of a purely stochastic process, but rather a reversion to a phylogenetically prior state. The single-celled eukaryotes from which multicelled organisms evolved needed to be able to survive and proliferate rapidly, especially during times of stress. During the transition to multicellularity, this proliferative capacity needed to be controlled, so that cells would work for the good of whole rather than pursuing their own inter-

\footnotetext{
${ }^{26}$ Thus for example Buss ([1987]) writes: 'the organism is an environment potentially populated by normal and variant cells. The variant must complete with the lineage from which it arose for growth-enhancing substances and, ultimately, for access to the germ line' (p. 76). Here Buss moves seamlessly between the two ideas.
} 
ests. The control mechanism that evolved involved suppressing, rather than eliminating altogether, the capacity for unlimited proliferation; and so the cells of modern organisms retain that capacity. Cancer is what occurs when the organism's system of networked control fails, often as a result of mutations to tumour suppressor genes, and thus represents a kind of atavistic reversion to a unicellular mode of life.

The atavistic hypothesis, first advanced by Davies and Lineweaver ([2011]), is supported by an impressive array of evidence. Many of the hallmarks of cancer, such as escape from apoptotic control, resistance to signalling, and uncontrolled proliferation, make sense once we think of them as cellular phenotypes that would have been adaptive for our single-celled ancestors (Greaves [2015]); and these phenotypes would have required suppression in order for cohesive multicelled organisms to evolve. Moreover, there is evidence that the genes that are implicated in causing cancer today have phlyogenetic origins that are associated with the initial emergence of multicellularity (Cisneros et al. [2017]); this suggests that these genes evolved to facilitate cellular cooperation, and organismic control over cell division, during the transition from single to multi-celled life. Finally, recent molecular studies have found that in many tumours, genes of known unicellular origin are preferentially expressed vis-à-vis genes of metazoan origin (Trigos et al. [2017]); this too supports the idea that cancerous cells exhibit phenotypes characteristic of the unicellular ancestors of the metazoa.

The atavistic hypothesis, as understood here, involves two claims. The first is that cancer cells express phenotypes characteristic of our single-celled ancestors; the second is that multicellular organisms evolved control mechanisms whose function it was to suppress these phenotypes, and whose breakdown leads to cancer today. These two claims are logically separable; that cancer cells exhibit phenotypic reversion does not necessarily imply that there was direct selection for suppression of the phenotypic behaviours in question. $^{27}$

The atavistic hypothesis, if true, furnishes a plausible response to the EDA. To see this, note firstly that it is fairly clear, and widely accepted, that the evolutionary transition from unicellularity to multicellularity involved conflict between levels of selection. ${ }^{28}$ That is, the transition required that

\footnotetext{
${ }^{27} \mathrm{An}$ anonymous referee notes that a single-celled bottleneck (if present) may have been largely sufficient to limit cell-organism conflicts during the evolution of multicellularity, but may have evolved for reasons unrelated to conflict-suppression.

${ }^{28}$ For discussion of how the levels of selection issue relates to evolutionary transitions,
} 
free-living or 'selfish' cells sacrifice their individuality and become part of the collective; and this required that cells' activities, including the process of cell division, be brought under organismic control. Thus for the transition to occur, organism-level selection had to trump cell-level selection; and cells had to evolve cooperative behaviours.

Now when this well-known point is combined with the atavistic hypothesis, a rationale emerges for conceptualizing cancer in terms of evolutionary conflict, despite the fact that cancer cells are not transmitted. For if cancer involves a reversion to a unicellular mode of life, as per the atavistic hypothesis, then it follows that cancer cells exhibit the very selfish phenotypes that had to be suppressed in order for metazoa to evolve; and whose suppression involved selection at the organism level trumping selection at the cell level. Therefore, although a cancer cell enjoys only a short-term proliferative advantage, the cellular mechanisms it employs to reap that advantage are similar to those found in its unicelled ancestors (and may depend on homologous genes). These unicelled ancestors were selfish in the literal sense, and their selfishness had to be curtailed in order for cohesive multicelled organisms to evolve. Thus there is a justification for regarding a cancer cell in a modern organism as 'cheating', undermining the collective for its own benefit, despite being an evolutionary dead-end; since its phenotypic characteristics are objectively similar to those of its unicellular ancestors who really did behave selfishly, and whose selfish tendencies were suppresed in the transition to multicellularity.

The atavistic hypothesis thus furnishes us with a principled reason for applying concepts and descriptors from social evolution theory to cancer, and thus constitutes a response to the EDA. It is a stronger response that the Buss-inspired response of the previous subsection, for two reasons. Firstly, the atavistic hypothesis arguably enjoys stronger empirical support than Buss's hypothesis. Secondly, there is one aspect of the EDA that the Bussinspired response does not speak to but the atavistic idea does. This is the analogy between organism's anti-cancer adaptations and the policing mechanisms found in social insect colonies.

To see why, note that if Buss's hypothesis is true, then germ-soma sequestration certainly counts as a suppression mechanism designed to limit the impact of rogue somatic cells. However, the analogy that Shpak and Lu

see (Maynard Smith [1989]; Michod [1999]; Okasha [2005]; Godfrey-Smith [2009]; Birch $[2018])$. 
([2016]) reject concerns organismic features such as apoptosis and immune system attack on nascent tumours - it is these, rather than germ-line sequestration, that are often analogized to worker policing in social insects. But Buss's hypothesis does not vindicate this analogy. Worker policing is a means by which worker sterility is enforced, and thus by which the colony-level analogue of germ-soma division - reproductive division-of-labour between queen and workers - is maintained. But apoptosis (for example) is not a means for reinforcing germ-soma division in metazoa, but rather a means for preventing cells engaging in unlimited proliferation to the detriment of the organism. That is, the function (or a function) of apoptosis is to prevent rogue somatic cells from harming the organism, but not necessarily to prevent them from invading the germ-line. Thus the analogy between apoptosis and worker policing does not go through, even supposing the truth of Buss's hypothesis.

If the atavistic hypothesis is true, however, then the analogy is defensible. For the various control mechanisms that organisms use to regulate celldivision (such as apoptosis), and whose breakdown leads to cancer, can then be seen as adaptations for suppressing cellular phenotypes that are detrimental to the collective, but that would have been beneficial to the unicellular ancestors in which those phenotypes first arose. And this is precisely the functional role that mechanisms such as worker policing play in social insect colonies: the colony needs to suppress the behaviour of workers, such as their egg-laying tendencies, in order for colony benefit to be optimized. So on the atavistic hypothesis, there is an isomorphism of functional role between at least some of an organism's anti-cancer adaptations and the policing mechanisms found in social insect colonies. By contrast, on the Buss hypothesis, this analogy is a weak one, given that the function of anti-cancer adaptations is not to protect the organism against germ-line invasion.

\section{Conclusion}

Over the last three decades, evolutionists have come to realize that the core themes of social evolution theory, such as cooperation versus conflict, kinship and multilevel selection, have a wider domain of application than was originally envisaged. Whenever there are fitness-affecting interactions between biological units, and whenever smaller units are nested within larger ones, these themes potentially become relevant. In particular, social evolution theory has proved indispensable for understanding how biological collectives, 
including chromosomes, eukaroytic cells, insect colonies and multicelled organisms, evolved in the first place, and how their cohesiveness is maintained given the potential conflicts of interest among their constituent sub-units. ${ }^{29}$

The generality and reach of social evolution theory is undeniably impressive. But there is an attendant risk, namely that certain biological phenomena are viewed through the lens of social evolution when they should not be. This is not merely a hypothetical possibility. In an article entitled 'Microbes are not bound by sociobiology', Rainey et al. ([2013]) have argued persuasively that terms and concepts from social evolution theory, such as 'cheating' and 'public good', have been misappropriated in studies of bacterial sociality, with harmful consequences. In particular, Rainey et al. object to the practice of using the term 'public good' to refer to any extracellular metabolite secreted by a microbe, irrespective of whether it is actually costly to produce or beneficial to others. An a priori commitment to the social evolution framework appears to be the source of this confusing practice.

The issue we have examined in this paper is similar, in that it centers on whether cancer should be viewed through the social evolution lens, and in particular on whether cancer is correctly conceptualized as a conflict between levels of selection. As noted in section 1, many cancer researchers do think and talk this way. However, the EDA as outlined by Gardner ([2015a]) and Shpak and Lu ([2016]), plus the supporting considerations introduced in section 3, pose a powerful challenge to this practice. This challenge cannot be met simply by appeal to the clonal evolution model of cancer, nor by invoking the fact that cancer cell lineages proliferate faster than normal somatic ones. However, in section 4 we examined an alternative justification for regarding cancer cells as selfish cheats benefiting themselves at the expense of the organism, that derives from facts (or assumptions) about the deep evolutionary past. Two versions of this argument were explored, one stemming from Buss's theory on the evolution of multicellularity, the other from the cancer-as-atavism hypothesis. In prinicple, either version permits a well-motivated response to the EDA, contingent on the relevant empirical facts being true. However, the atavistic hypothesis appears more promising.

Finally, the methodology employed in this paper deserves brief comment. We began by noting that cancer is often viewed as a conflict between selective levels, and the objection to this posed by the EDA. We then asked

\footnotetext{
${ }^{29}$ See, for example, (Queller and Strassmann [2009]; Bourke [2011]; Maynard Smith [1989]; Birch [2018]).
} 
what the world would have to be like in order for this view of cancer to be defensible. In effect, then, our enquiry has sought to determine the empirical preconditions that would have to be met in order to justify a certain way of describing, explaining and conceptualizing a particular phenomenon (cancer). These preconditions are not self-evident, but took some work to uncover. I suggest that the same may be true in other cases. Examining the empirical preconditions of a particular area of scientific discourse, or a particular explanatory practice, may be a fruitful technique for philosophers of science to use, especially when the discourse or explanatory practice is contested.

\section{Acknowledgements}

For comments and discussion, thanks to Lucie Laplane, Anya Plutynski, Thomas Pradeu, Bertrand Daignan-Fornier, Andy Gardner and to three anonymous referees for $B J P S$. This paper is part of a project that has received funding from the European Research Council (ERC) under the European Union's Horizon 2020 research and innovation programme (grant agreement number 101018533).

Samir Okasha

Department of Philosophy

University of Bristol, U.K.

Samir.Okasha@bristol.ac.uk 


\section{References}

Aktipis, A. [2020]: The Cheating Cell, Princeton: Princeton University Press.

Aktipis, C. A., Boddy, A. M., Jansen, G., Hibne, U., Hochberg, M. E., Maley, C. C. and Wilkinson, G. S. [2015]: 'Cancer Across the Tree of Life: Cooperation and Cheating in Multicellularity', Philosophical Transactions of the Royal Society B, 370, p. 20140219.

Aktipis, C. A. and Maley, C. C. [2017]: 'Cooperation and Cheating as Innovation: Insights from Cellular Societies', Philosophical Transactions of the Royal Society of London B, 372, p. 20160421.

Aktipis, C. A. and Nesse, R. M. [2013]: 'Evolutionary Foundations for Cancer Biology', Evolutionary Applications, 6, pp. 144-59.

Arnold, A. J. and Fristrup, K. [1982]: 'The Theory of Evolution by Natural Selection: a Hierarchical Expansion', Paleobiology, 8, pp. 113-29.

Axelrod, R., Axelrod, D .E. and Pienta, K. J. [2006]: 'Evolution of Cooperation Among Tumour Cells', Proceedings of the National Academy of Sciences, 103, pp. 13474-9.

Birch, J. [2018]: The Philosophy of Social Evolution, Oxford: Oxford University Press.

Bourke, A. F. G. [2011]: Principles of Social Evolution, Oxford: Oxford University Press.

Boveri, T. [1914]: The Origin of Malignant Tumours, London: Baillière, Tindall and Cox.

Brunet, T. D. P. and Doolittle, W. F. [2015]: 'Multilevel Selection Theory and the Evolutionary Functions of Transposable Elements', Genome Biology and Evolution, 7, pp. 2445-57.

Burt, A. and Trivers, R. [2006]: Genes in Conflict, Harvard: Harvard University Press.

Buss, L. [1987]: The Evolution of Individuality, Princeton: Princeton University Press. 
Cairns J. [1975]: 'Mutation, Selection and the Natural History of Cancer', Nature, 255, pp. 197-200.

Cisneros, L., Bussey, K. J., Orr A. J., Miočević, M, Lineweaver, C. H. and Davies, P. [2017]: 'Ancient Genes Establish Stress-Induced Mutation as a Hallmark of Cancer', PLOS One, 12, p. e0176258.

Claveria, C. and Torres, M. [2016]: 'Cell Competition: Mechanisms and Physiological Roles', Annual Review of Cell and Developmental Biology, 32, pp. 411-39.

Cleary, A. S., Leonard T. L., Gestl, S. A. and Gunther, E. J. [2014]: 'Tumour Cell Heterogeneity Maintained by Cooperating Subclones in Wnt-Driven Mammary Cancers', Nature, 508, pp. 113-7.

Crespi, B. and Summers, B. [2005]: 'Evolutionary Biology of Cancer', Trends in Ecology and Evolution, 2, pp. 545-52.

Damuth J. and Heisler I. L. [1988]: 'Alternative Formulations of Multilevel Selection', Biology and Philosophy, 3, pp. 407-30.

Davies, P. C. W. and Lineweaver, C. H. [2011]: 'Cancer Tumours as Metazoa 1.0: Tapping Genes of Ancient Ancestors', Physical Biology, 8, p. 015001.

Fisher, R. A. [1930]: The Genetical Theory of Natural Selection, Oxford: Clarendon Press.

Fortunato A., Boddy A., Mallo D., Aktipis A., Maley, C. C. and Pepper, J. W. [2017]: 'Natural Selection in Cancer Biology: from Molecular Snowflakes to Trait Hallmarks', Cold Spring Harbour Perspectives in Medicine, 7, p. a029652.

Frank, S. A. [1998]: Foundations of Social Evolution, Princeton: Princeton University Press.

Gardner, A. [2015a]: 'The Genetical Theory of Multilevel Selection', Journal of Evolutionary Biology, 28, pp. 305-19.

Gardner, A. [2015b]: 'More on the Genetical Theory of Multilevel Selection', Journal of Evolutionary Biology, 28, pp. 1747-51. 
Germain, P. [2012]: 'Cancer Cells and Adaptive Explanations', Biology and Philosophy, 27, pp. 785-810.

Germain, P. and Laplane, L. [2017]: 'Metastasis as Supra-Cellular Selection? A Reply to Lean and Plutynski', Biology and Philosophy, 32, pp. 281-7.

Godfrey-Smith, P. [2009]: Darwinian Populations and Natural Selection, Oxford: Oxford University Press.

Goodnight, C. J. [2013]: 'On Multilevel Selection and Kin Selection: Contextual Analysis Meets Direct Fitness', Evolution, 67, pp. 1539-48.

Goodnight, C. J. [2015]: 'Multilevel Selection Theory and Evidence: a Critique of Gardner 2015', Journal of Evolutionary Biology, 28, pp. 1734-46.

Gould, S. J. [2002]: The Structure of Evolutionary Theory, Harvard: Harvard University Press.

Greaves, M. [2015]: 'Evolutionary Determinants of Cancer', Cancer Discovery, 5, pp. 806-20.

Hanahan D. and Weinberg R. A. [2000]: 'The Hallmarks of Cancer', Cell, 100, pp. 57-70.

Hanahan, D. and Weinberg R. A. [2011]: 'Hallmarks of Cancer: The Next Generation', Cell, 144, pp. 646-74.

Inglis, R. F., Ryu, E., Asikhia, O., Strassmann, J. E. and Queller, D. C. [2017]: 'Does High Relatedness Promote Cheater-Free Multicellularity in Synthetic Lifecycles?', Journal of Evolutionary Biology, 30, pp. 985-93.

Lanfear, R. [2018]: 'Do Plants Have a Segregated Germline?', PLOS Biology, 16, p. e2005439.

Laplane, L. [2018]: 'Cancer Stem Cells Modulate Patterns and Processes of Evolution in Cancers', Biology and Philosophy, 33, 18.

Lean, C. and Plutynski, A. [2016]: 'The Evolution of Failure (Reply to Germain)', Biology and Philosophy, 31, pp. 39-57.

Leija-Salazar, M., Piette, C. L. and Proukakis, C. [2018]: 'Somatic Mutations in Neurodegeneration', Neuropathology and Applied Neurobiology, 44, pp. $267-85$. 
Lewontin, R. C. and Dunn, L. C. [1960]: 'The Evolutionary Dynamics of a Polymorphism in the House Mouse', Genetics, 45, pp. 705-22.

Li, C. and Williams, S. M. [2013]: 'Human Somatic Variation: It's Not Just For Cancer Anymore', Current Genetic Medicine Reports, 1, pp. 212-18.

Maley, C. C. and Reid, B. J. [2005]: 'Natural Selection in Neoplastic Progression of Barrett's Esophagus', Seminars in Cancer Biology, 15, pp. 474-83.

Maynard Smith, J. [1989]: 'Evolutionary Progress and the Levels of Selection', in M. H. Nitecki (ed), Evolutionary Progress, Chicago: University of Chicago Press, pp. 219-230.

Maynard Smith, J. and Szathmáry, E. [1995]: The Major Transitions in Evolution, Oxford: Oxford University Press.

Merlo L. M., Pepper J. W., Reid B. J. and Maley C. C. [2006]: 'Cancer as an Evolutionary and Ecological Process', Nature Reviews Cancer, 6, pp. 924-35.

Michod, R. E. [1999]: Darwinian Dynamics: Evolutionary Transitions in Fitness and Individuality, Princeton: Princeton University Press.

Michod, R. E. [2007]: 'Evolution of Individuality During the Transition From Unicellular to Multicellular Life', Proceedings of the National Academy of Sciences USA, 104, pp. 8613-8.

Michod, R. E. and Roze, D. [1999]: 'Cooperation and Conflict in the Evolution of Individuality. III. Transitions in the Unit of Fitness', in C. L. Nehaniv (ed), Mathematical and Computational Biology: Computational Morphogenesis, Hierarchical Complexity, and Digital Evolution, Providence, R. I.: American Mathematical Society, pp. 47-91.

Morange, M. [2012]: 'What History Tells Us XXVIII. What is Really New in the Current Evolutionary Theory of Cancer?' Journal of Biosciences, 37, pp. 609-12.

Moreno, E. and Rhiner, C. [2014]: 'Darwin's Multicellularity: from Neurotrophic Theories and Cell Competition to Fitness Fingerprints', Current Opinion in Cell Biology, 31, pp. 16-22. 
Murchison, E. [2008]: 'Clonally Transmissible Cancers in Dogs and Tasmanian Devils', Oncogene, 27, pp. S19-S30.

Nelson, P. and Masel, J. [2017]: 'Intercellular Competition and the Inevitability of Multicellular Aging', Proceedings of the National Academy of Sciences USA, 114, pp. 12982-7.

Nowell, P. C. [1976]: 'The Clonal Evolution of Tumour Cell Populations', Science, 194, pp. 23-8.

Okasha, S. [2005]: 'Multilevel Selection and the Major Transitions in Evolution,' Philosophy of Science, 72, pp. 1013-25.

Okasha, S. [2006]: Evolution and the Levels of Selection, Oxford: Oxford University Press.

Plutynski, A. [2018]: Explaining Cancer, Oxford: Oxford University Press.

Plutynski, A. [2020]: 'Cancer Modeling: the Advantages and Limitations of Multiple Perspectives', in M. Massimi, and C. D. McCoy (eds), Understanding Perspectivism, Abingdon: Taylor and Francis, pp. 160-77.

Price, G. R. [1972]: 'Extension of Covariance Selection Mathematics', Annals of Human Genetics, 35, pp. 485-90.

Queller D. C. [2000]: 'Relatedness and the Fraternal Major Transitions', Philosophical Transactions of the Royal Society B, 355, pp. 1647-55.

Queller, D. C. and Strassmann, J. E. [2009]: 'Beyond Society: the Evolution of Organismality', Philosophical Transactions of the Royal Society B, 364, pp. 3143-55.

Queller, D. C. and Strassmann, J. E. [2013]: 'Experimental Evolution of Multicellularity Using Microbial Pseudo-Organisms', Biology Letters, 9, p. 20120636.

Rainey, P. B., Desprat, N., Driscoll, W. W., and Zhang, X. X. [2014]: 'Microbes Are Not Bound By Sociobiology: Response to Kümmerli and RossGillespie (2013)', Evolution, 68, pp. 3344-55. 
Ratcliff, W. C., Ford Denison, R., Borrello, M. and Travisano, M. [2012]: 'Experimental Evolution of Multicellularity', Proceedings of the National Academy of Sciences USA, 109, pp. 1595-600.

Schwartz R. and Schäffer, A. A. [2017]: 'The Evolution of Tumour Phylogenetics: Principles and Practice', Nature Reviews Genetics, 18, pp. 213-29.

Shpak, M. and Lu, J. [2016]: 'An Evolutionary Genetic Perspective on Cancer Biology', Annual Review of Ecology, Evolution and Systematics, 47, pp. 25-49.

Sober, E. and Wilson, D. S. [1998]: Unto Others: The Evolution and Psychology of Unselfish Behaviour, Harvard: Harvard University Press.

Trigos, A. S., Pearson, R. B., Papenfuss, A. T. and Goode, D. L. [2017]: 'Altered Interactions Between Unicellular and Multicellular Genes Drive Hallmarks of Transformation in a Diverse Range of Solid Tumours', Proceedings of the National Academy of Sciences USA, 114, pp. 6406-11.

Vincent, M. D. [2010]: 'The Animal Within: Carcinogenesis and the Clonal Evolution of Cancer Cells are Speciation Events Sensu Stricto', Evolution, 64, pp. 1173-83.

Werner B. and Sottoriva A. [2018]: 'Variation of Mutational Burden in Healthy Human Tissues Suggests Non-Random Strand Segregation and Allows Measuring Somatic Mutation Rates', PLOS Computational Biology, 14, p. e1006233. 